\title{
Observations of Traveling Crossflow Resonant Triad Interactions on a Swept Wing
}

\author{
Jenna Eppink ${ }^{*}$ \\ NASA Langley Research Center, Hampton, VA, 23681 \\ and \\ Richard Wlezien ${ }^{\dagger}$ \\ Iowa State University, Ames, Iowa, 50011
}

\begin{abstract}
Experimental evidence indicates the presence of a triad resonance interaction between traveling crossflow modes in a swept wing flow. Results indicate that this interaction occurs when the stationary and traveling crossflow modes have similar and relatively low amplitudes $(\sim 1 \%$ to $6 \%$ of the total freestream velocity). The resonant interaction occurs at instability amplitudes well below those typically known to cause transition, yet transition is observed to occur just downstream of the resonance. In each case, two primary linearly unstable traveling crossflow modes are nonlinearly coupled to a higher frequency linearly stable mode at the sum of their frequencies. The higher-frequency mode is linearly stable and presumed to exist as a consequence of the interaction of the two primary modes. Autoand cross-bicoherence are used to determine the extent of phase-matching between the modes, and wavenumber matching confirms the triad resonant nature of the interaction. The bicoherence results indicate a spectral broadening mechanism and the potential path to early transition. The implications for laminar flow control in swept wing flows are significant. Even if stationary crossflow modes remain subcritical, traveling crossflow interactions can lead to early transition.
\end{abstract}

\section{Nomenclature}

$\begin{array}{ll}b & =\text { bicoherence } \\ C_{p} & =\text { pressure coefficient } \\ C_{q} & =\text { suction coefficient } \\ \mathrm{c} & =\text { normal chord } \\ f & =\text { frequency } \\ k & =\text { stationary mode spanwise wavenumber } \\ R e & =\text { Reynolds number } \\ |\bar{u}| & =\text { stationary disturbance velocity amplitude } \\ \bar{u} & =\text { mean velocity } \\ u & =\text { rms disturbance velocity } \\ U_{\infty} & =\text { freestream velocity } \\ v_{p} & =\text { spanwise phase velocity } \\ x & =\text { chordwise direction } \\ y & =\text { wall-normal direction } \\ z & =\text { spanwise direction } \\ \lambda & =\text { spanwise wavelength } \\ \theta & =\text { ensemble averaged phase }\end{array}$

\footnotetext{
* Graduate Student Trainee, Computational AeroSciences Branch, Mail Stop 170, AIAA Student Member

${ }^{\dagger}$ Professor and Vance and Arlene Coffman Endowed Department Chair in Aerospace Engineering, 2271 Howe Hall, Room 1200A, AIAA Fellow
} 


\section{Background}

$\mathrm{T}$ HE path to transition in two-dimensional flows is fairly well understood. It is generally accepted that, in low disturbance environments, transition to turbulence occurs through the growth of linear Tollmien-Schlichting (TS) instabilities. These instabilities, once introduced into the boundary layer (via receptivity mechanisms), experience a period of exponential growth, followed by nonlinear interactions and breakdown to turbulence. Depending on the amplitude of the T-S waves (determined by the freestream disturbance level), three different types of breakdown have been identified: C-type, H-type, and K-type. The K-type breakdown, first observed by Klebanoff $^{1}$, is characterized by a lambda-type structure which appears when the rms amplitude of the T-S disturbances approach $1 \%$ of the freestream velocity $\left(\mathrm{U}_{\infty}\right)$. This type of breakdown is characterized by threedimensionality, followed by high frequency spikes and spectral broadening. For T-S amplitudes between $0.3 \%$ to $0.6 \%$ of $U_{\infty}$ subharmonic breakdown occurs. Two distinct subharmonic breakdown mechanisms have been discovered; these have become known as C-type and H-type breakdown.

The subharmonic route to turbulence was first proposed by $\mathrm{Craik}^{2}$, who studied a resonant triad interaction. This breakdown mechanism, known as C-type breakdown, involves the interaction of a two-dimensional T-S wave with two symmetric oblique waves. The interaction is expected to be quite strong due to the phase-coupling between the modes and the potential energy transfer from the primary shear flow to the disturbance in the vicinity of the critical layer. Resonance occurs when the phase speeds of the three wave components match. This mechanism provides a means by which a pair of oblique waves may be amplified even when linear stability theory predicts that they are damped. The second type of subharmonic breakdown, known as H-type breakdown, was analyzed by Herbert ${ }^{3}$ and involves a secondary instability of the streamwise periodic flow to three-dimensional disturbances.

$\mathrm{Saric}^{4}$ performed flow visualization experiments to investigate the subharmonic route to turbulence and found that at intermediate T-S disturbance amplitudes (between 0.3 and $0.6 \% \mathrm{U}_{\infty}$ at Branch II) H-type breakdown occurred, while at lower amplitudes between $0.2 \%$ and $0.3 \%$, C-type breakdown was observed. At amplitudes greater than $0.6 \%$, K-type breakdown occurred.

Lekoudis ${ }^{5}$ considered the possibility of a resonant triad interaction between traveling crossflow waves in the three-dimensional flow on a swept wing. He found that it is indeed possible for this type of interaction to exist, and he noted several important differences from the two-dimensional case. Most noteably, it is possible for this mechanism to exist for three linearly unstable modes, which could make the interaction much stronger. There is also no need for the waves to satisfy the symmetry condition, nor for the phase speeds to match.

Malik et al. ${ }^{6}$ studied the nonlinear interaction of traveling and stationary crossflow modes. They only considered tuned cases, for which the two traveling crossflow modes had equal and opposite spanwise wavenumbers and equal frequencies. They found that a resonant triad type interaction could exist, depending on the relative initial amplitudes of the disturbances. When the initial amplitude of the stationary mode is large compared to the traveling mode, the stationary vortex dominates most of the downstream development. But, owing to the larger growth rate of the traveling modes, at some point the traveling mode reaches comparable amplitudes with the stationary mode. At this point, interaction of the traveling mode with the harmonic of the stationary mode leads to the amplification of another traveling mode with the same frequency and equal and opposite wavenumber, and a resonant triad is set up.

Corke \& Knasiak $^{7}$ found evidence of a resonant triad interaction in a three-dimensional boundary layer on a rotating disk. They observed the interaction of two traveling crossflow modes with a lower amplitude lowwavenumber stationary mode, which emerged only in the nonlinear region. They verified a triple phase-locking between the three modes, as well as wavenumber matching. They also found that as the Reynolds number was increased, the number of traveling modes which were in resonance with the stationary crossflow mode increased and spread to higher frequencies.

Dagenhart et al. ${ }^{8}$, under similar circumstances to the present experiment (swept wing with natural transition occurring far downstream - around $\mathrm{x} / \mathrm{c}=0.6$, and with measurements taken close to the transition location), observed a broad peak in the hot-film spectrum in a higher frequency range than the predicted amplified band for traveling crossflow. They concluded that these disturbances might be harmonics arising due to a non-linear parametric resonance with the primary mode.

In this paper we present evidence and discuss implications of a triad resonance interaction that was observed in the Hybrid Laminar Flow Control (HLFC) Crossflow Experiment. The triad resonance occurred between three traveling crossflow modes and was observed just upstream of transition. The existence of a resonant triad interaction in swept wing flows would have important implications for wind tunnel laminar flow testing as well as laminar flow control technology.

Most transition and stability research for swept-wing flows has been aimed at understanding stationary crossflow, since this is the type of disturbance that is expected to dominate in flight due to low disturbance levels 
experienced in that environment. However, it is possible for low amplitude traveling crossflow modes to interact and amplify, even if the stationary crossflow modes are successfully damped. In this case, the traveling crossflow modes may still lead to transition. It is important to further understand this path to breakdown and the factors that lead to the presence of triad resonant interactions.

\section{The Hybrid Laminar Flow Control Experiment}

The NASA/Boeing Hybrid Laminar Flow Control (HLFC) Experiment was an ambitious fundamental transition experiment performed in the NASA Langley 8-ft. Transonic Pressure Tunnel (TPT). The result was a large and unique set of data. The test was designed over a two-year period, and the testing, which took over a year, was completed in December 1995. Wlezien gives a historical overview of the test, and the then state of the art technologies which were developed to make the test a successful collaboration between NASA and Boeing. Budget reductions in the late 1990s ended all analysis of the data, but a request was initiated in 2008 to restore the HLFC database to complete the data analysis.

The purpose of this experiment was the study of the effect of suction on the transition process in swept wing flows. The model used in the HLFC experiment was a 3-m streamwise chord, 35-degree swept wing. The model is shown inside of the test section in Fig. 1, where the traverse system is visible towards the back of the wing. The traverse system was moved manually to each of the desired chord locations, and could be controlled remotely to traverse in the spanwise direction. Four interchangeable, identically contoured leading edge panels were used to examine the effects of different suction distributions. Three of the panels were perforated, with hole patterns designed to answer specific questions, and the fourth was a solid panel. The leading edge panel made up the first $20 \%$ of the wing, and needed to be joined smoothly to the midchord region of the wing. This joint location is evident in Fig. 1 as a vertical white line, and this area in particular required a significant amount of work during the experiment to ensure that the flow was not tripped prematurely. Choudhari et al. ${ }^{10}$ and $\mathrm{HTC}^{11}$ provide more detailed descriptions of the experiment.

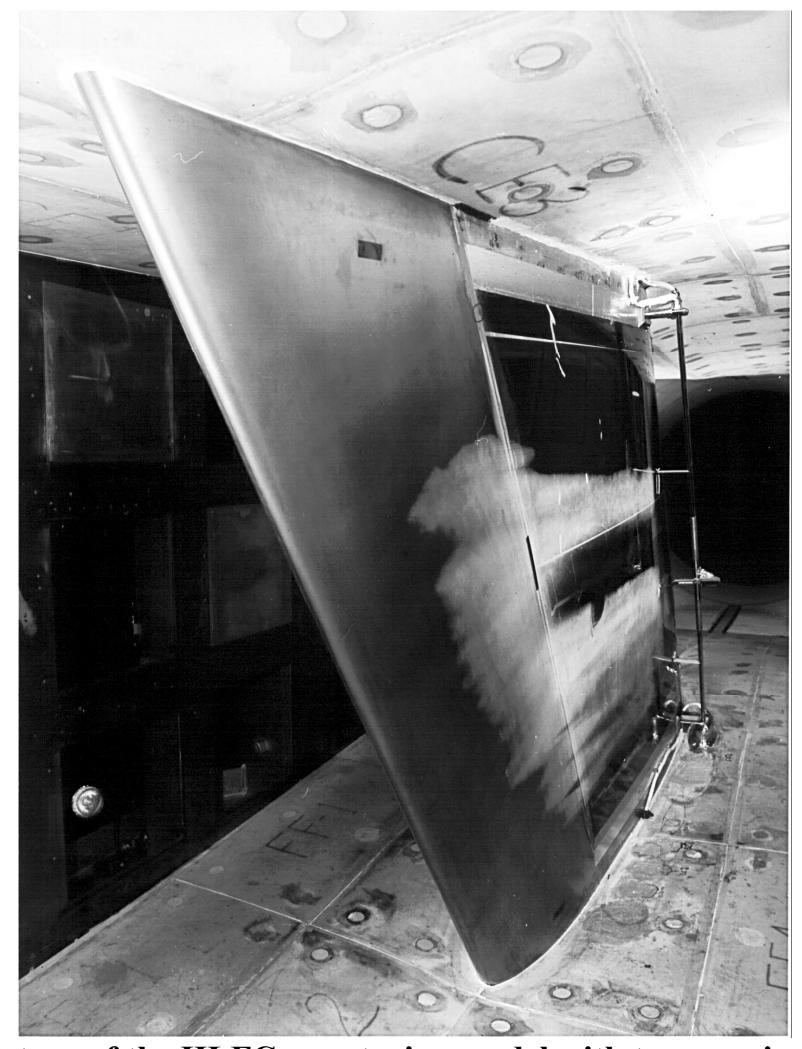

Figure 1. Picture of the HLFC swept wing model with traverse in the $8 \mathrm{ft}$. TPT

The tests were conducted at a Mach number of 0.24 and chord Reynolds numbers between 5 million and 25 million. The TPT was chosen for its low free-stream turbulence levels $(0.01$ to $0.02 \%)$ in this Reynolds number range. The airfoil shape was designed using CFD and stability prediction codes to obtain crossflow dominated 
transition. The airfoil was not symmetric and measurements were only performed on the upper surface, for which the shape and pressure distribution are shown in Fig. 2. Streett ${ }^{12}$ provides more information on the design of the airfoil shape. In order to avoid the growth of two-dimensional instabilities, the mid-chord region of the airfoil was cooled by circulating chilled water in channels under the airfoil surface.
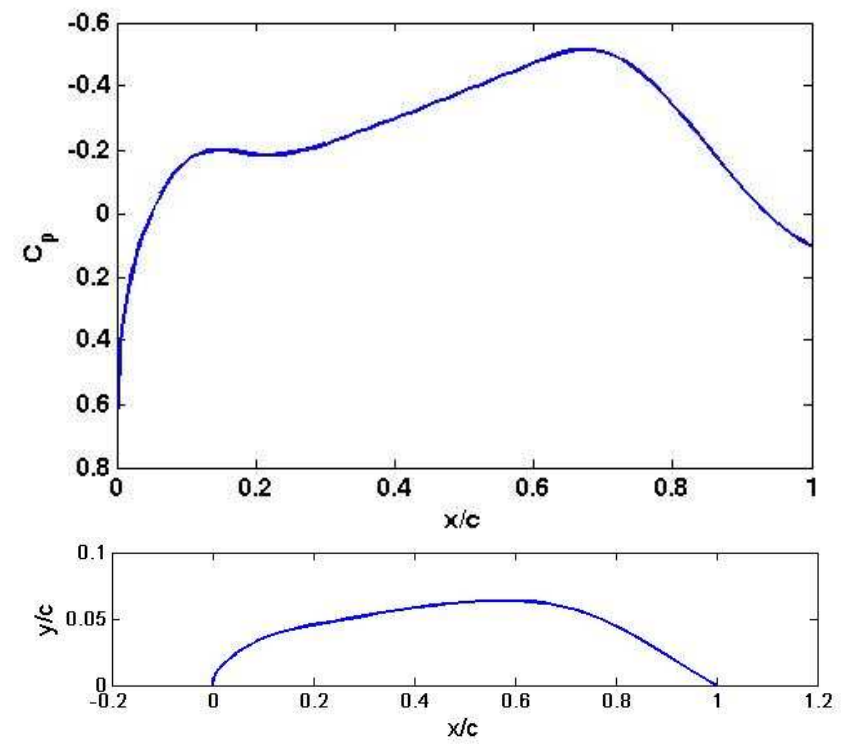

Figure 2. HLFC model shape and pressure distribution

The suction surfaces on leading edge panels 1 through 3 were divided into 20 individual chambers (called flutes), which spanned the first $20 \%$ chord of the airfoil (Fig. 3). Because the pressure in each flute was controlled separately, arbitrary suction distributions could be tested. The hole size, spacing, and row-to-row hole offset varied between panels. Panel 1 was designed as the most conservative case (i.e. most likely to delay transition), with the smallest sized holes and the finest spacing. Panel 2 was designed to study the detrimental effect of the suction hole pattern on transition. This panel thus had the largest hole size and spacing, and the hole pattern was roughly aligned with the direction of the inviscid streamline and spacing of highly-amplified crossflow modes. Panel 3 was meant to be representative of a practical design, with random row-to-row hole offsets and intermediate hole size and spacing. Panel 4 was a solid panel for investigating disturbance growth in the absence of suction.

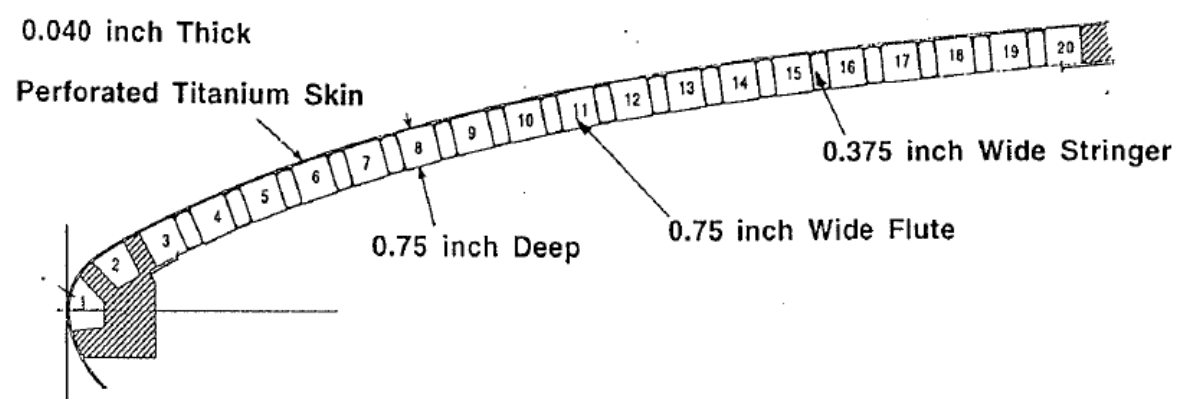

Figure 3. Cross section of leading edge panel showing flute arrangement

The data that were acquired consisted of mean surface pressures, infrared images, and hotwire measurements of steady and time-dependent velocities. A high resolution traversing mechanism was used to acquire boundary layer hotwire scans. Figure 4 shows the coordinate system relative to the wing. Two types of scans were taken: $y-z$ scans and z-scans. The y-z scans consisted of full boundary layer profiles spaced closely together in span in order to obtain a full 2D picture of the flowfield. Since the y-z scans proved to be quite time-consuming, a large portion of the data consists of z-scans, which were spanwise scans taken at selected heights above the surface. Dynamic data, sampled at $10 \mathrm{kHz}$, were taken for a limited number of cases of leading edge panel, suction distribution, and chord location. For these datasets, a fixed probe, located several millimeters away in span from the traverse probe at a 
constant wall-normal location, simultaneously acquired data. This data could then be used as a phase reference to calculate phase speeds. Transition detection was accomplished using surface heat flux measurements using an insulating surface film and an infrared camera.

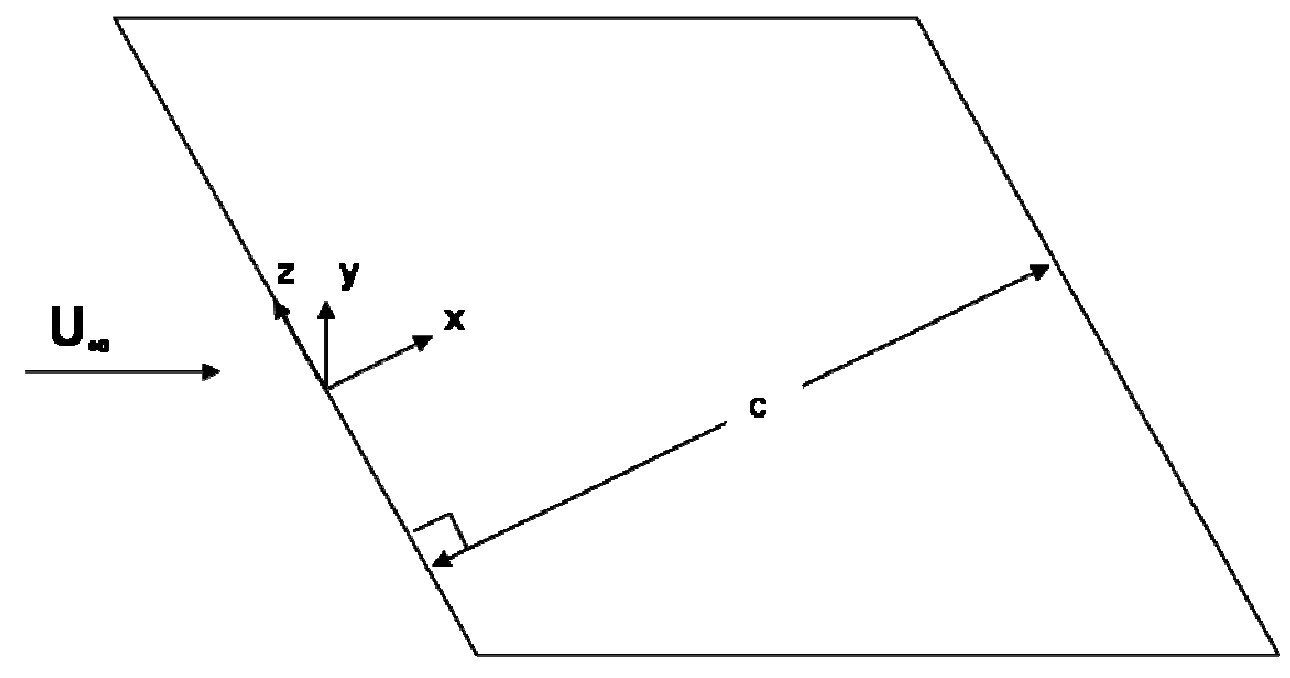

Figure 4. Coordinate system for swept wing

The details of the data analysis are described by Eppink and Wlezien ${ }^{13}$. The chordwise measurement locations were taken at only a limited number of points due to the necessity of manually relocating the traverse. Typically, for a given chordwise location, measurements were taken for several different conditions (i.e. Reynolds numbers and suction distributions) before moving the traverse to the next chordwise location.

The spanwise phase speeds for particular modes were calculated for the dynamic datasets as follows. The phase shift relative to the fixed probe was calculated throughout the portion of the boundary layer for which the two signals were highly coherent. This was performed for each spanwise location, so the result was 8-10 phase profiles. These profiles were then interpolated and averaged to obtain a spanwise average phase profile. For each spanwise location, the difference of the actual phase profile from the average phase profile was calculated, and then averaged across the boundary layer. Finally, a best fit line was applied to the data, and the slope of this line indicated the spanwise phase speed of the wave. The spanwise wavenumber was then calculated using the relation:

$$
k=\frac{f}{v_{p}}
$$

\section{Results}

\section{A. Overview}

The two data sets that will be discussed below were taken on leading edge panel 3 (the panel with intermediate hole size and spacing, and with random row offset) at a chordwise location of $\mathrm{x} / \mathrm{c}=0.52$. The flow conditions and transition locations obtained from the IR images are listed in Table 1. One can visually determine transition location from the IR images, but it is difficult to process the images to determine precise transition location. There is "smearing" of the perceived transition front due to thermal conductivity of the model, the threshold temperature at which transition is said to occur is somewhat arbitrary, and true transition is a somewhat arbitrary concept (i.e. is the temperature change indicative of the beginning of the transition region, the middle, or the end). We chose a threshold in the streamwise derivative of temperature as an indicator of transition. While a change in threshold value will result in a different transition location, the same criteria can be applied to each IR image. In this way the incremental difference in transition location from one case to the next can be as reliable as possible. Hence, the "incremental uncertainty," (Table 1) indicates the estimated uncertainty in the difference between the transition location from one case to the next, and results from the limited resolution of the image, as well as the variation in transition location across the measurement region. In both cases discussed in this paper the transition location was reasonably constant across the measurement region (i.e. no wedges), and the uncertainty was fairly low. The 
absolute uncertainty of the transition location is a bit more difficult to estimate, for all of the reasons described previously. However, if we simply look at the region over which the slope of the $\mathrm{x} v \mathrm{vs}$. intensity plot is large, we can develop a conservative estimate of the uncertainty. The range of transition location resulting from this analysis is also listed in Table 1 .

This data for both cases were obtained a few percent chord upstream of the transition location. The suction distributions for these cases are shown in Fig. 5. In both cases, the suction through flute 2 was decreased below the originally desired value due to problems with premature transition. Case 1 had overall greater suction than Case 2, which may account for the slightly later transition location and lower crossflow amplitudes.

Table 1. Test conditions and transition locations

\begin{tabular}{|c|c|c|c|c|c|}
\hline Case & $\begin{array}{c}\text { Unit Reynolds } \\
\text { number (M/ft) }\end{array}$ & $\begin{array}{c}\text { Chord Location } \\
(\mathbf{x} / \mathbf{c})\end{array}$ & $\begin{array}{c}\text { Transition Location } \\
\mathbf{( x / c )}\end{array}$ & $\begin{array}{c}\text { Total estimated } \\
\text { Incremental } \\
\text { Uncertainty (x/c) }\end{array}$ & $\begin{array}{c}\text { range of transition } \\
\text { location (x/c) }\end{array}$ \\
\hline 1 & 1.5 & 0.52 & 0.562 & \pm 0.010 & 0.56 to 0.60 \\
\hline 2 & 1.5 & 0.52 & 0.532 & \pm 0.007 & 0.53 to 0.57 \\
\hline
\end{tabular}

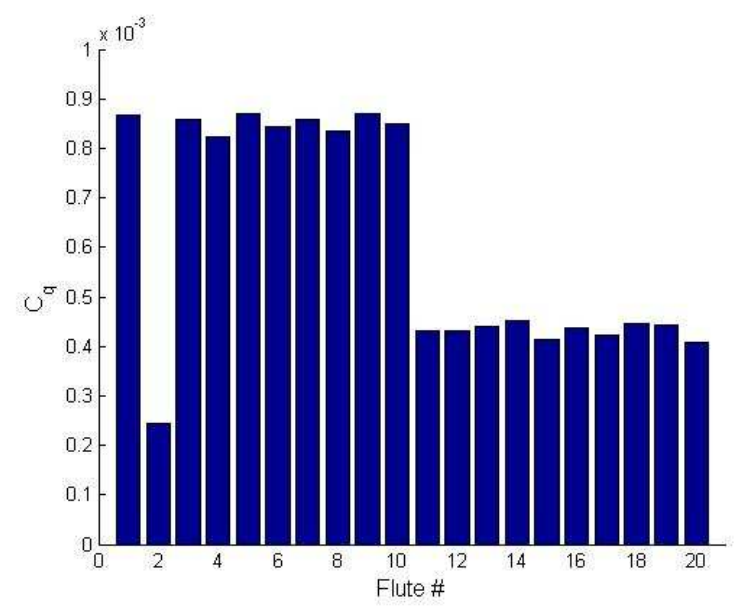

(a) Case 1

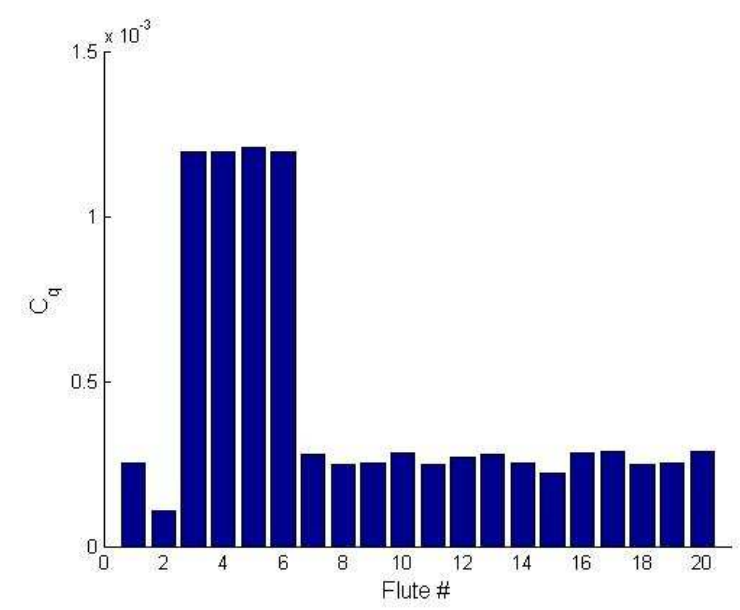

(b) Case 2

Figure 5. Suction distributions

The stationary crossflow amplitudes for these cases can be estimated from the spanwise hotwire scans that were taken at several different wall-normal locations. These are plotted in Figs.6-7, along with mode shapes calculated using the linear parabolized stability equations (LPSE) for comparison (these are scaled to match the measured amplitudes in a least-squares sense). There were two distinct peaks in the wavenumber spectra for Case 1, therefore two modes are plotted for that case. There is good agreement for the lower wavenumber mode for Case 1, as well as for the Case 2 mode. There is some discrepancy in the mode shape for the higher wavenumber mode for Case 1 (Fig. 6b). This could be due to nonlinear distortion of the mode due to interactions with the traveling crossflow, or it could simply be experimental error. Based on these results, the available data give a fairly reliable estimate of the stationary crossflow amplitudes. For these conditions, data were only obtained at a single chordwise location, therefore no growth rates can be calculated.

In both cases, the stationary crossflow amplitudes are low, despite the fact that the profiles are only $1-5 \%$ chord upstream of the transition location. For Case 1, the amplitudes of both modes are around $2 \%$ of $U_{\infty}$, while the amplitude of the mode in Case 2 is roughly $6 \%$ of $U_{\infty}$. Typically, in stationary crossflow dominated flows, stationary crossflow amplitudes are nearly $10-20 \%$ of $U_{\infty}$ at transition ${ }^{14-16}$. The dynamic hotwire data were investigated further to determine the cause of the seemingly early transition. These results are discussed in detail for each case. 


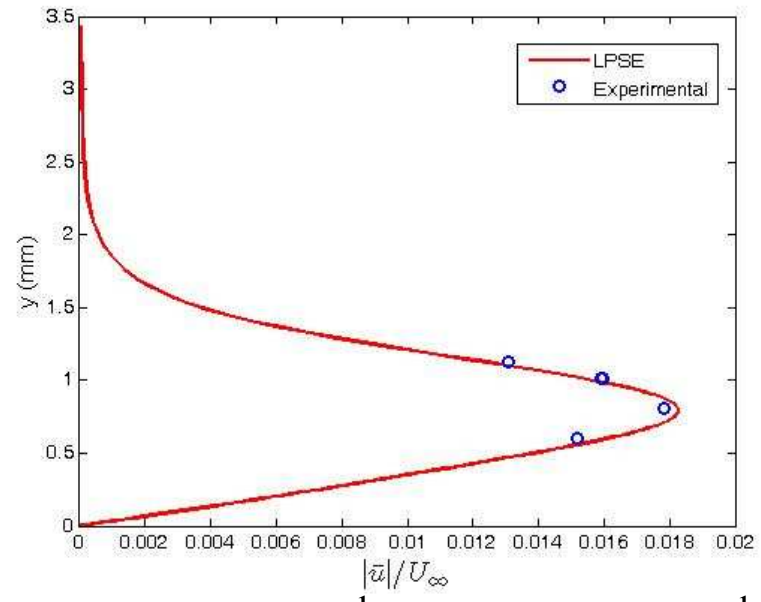

(a) LPSE: $\mathrm{k}=0.1 \mathrm{~mm}^{-1}$, Exp.: $\mathrm{k}=0.05$ to $0.1 \mathrm{~mm}^{-1}$

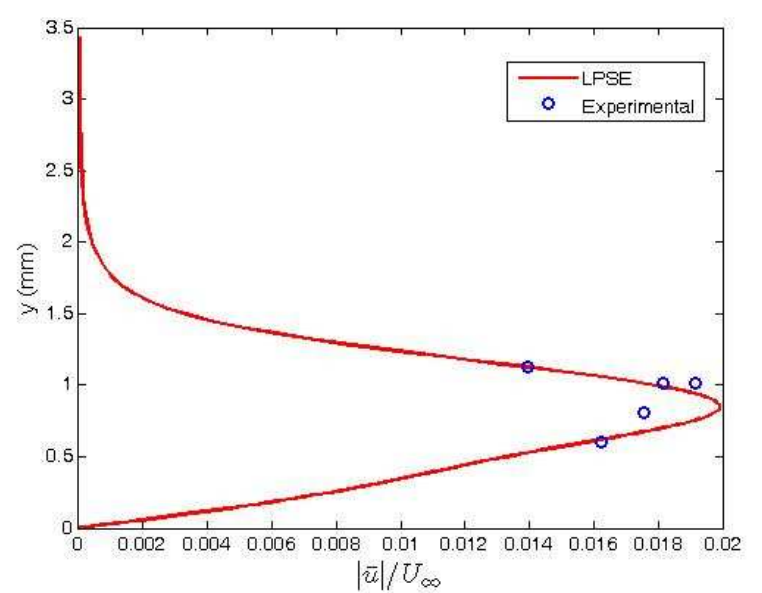

(b) LPSE: $\mathrm{k}=0.143 \mathrm{~mm}^{-1}$, Exp: $\mathrm{k}=0.1$ to $0.2 \mathrm{~mm}^{-1}$

Figure 6. Stationary crossflow mode shapes from Case 1

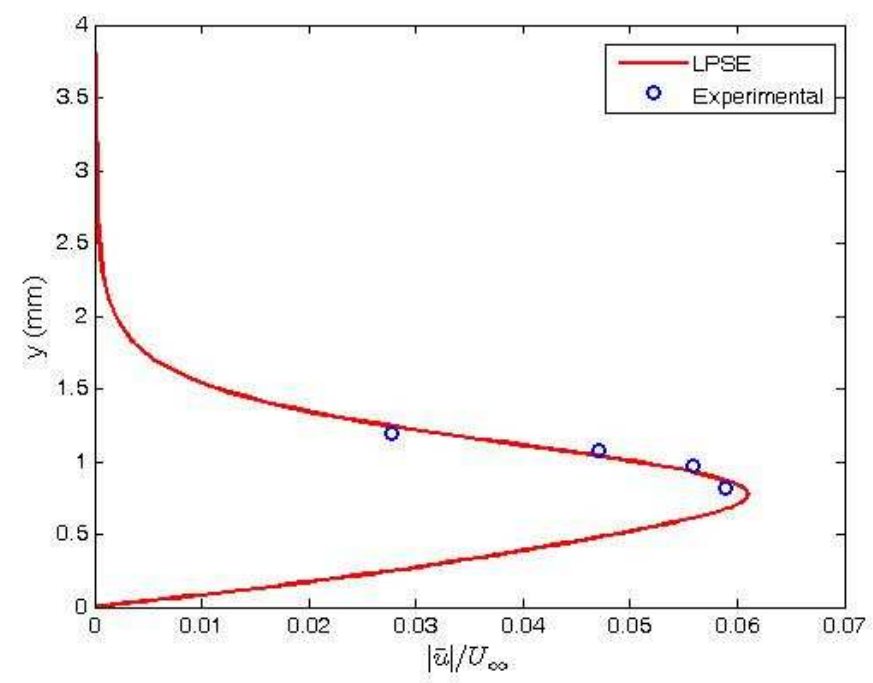

Figure 7. Stationary crossflow mode shape for Case 2. LPSE: $k=0.1 \mathrm{~mm}^{-1}$, Exp: $k=0.05$ to $0.2 \mathrm{~mm}^{-1}$

\section{B. Case 1}

\section{Traveling Crossflow}

A representative power spectrum of the raw hotwire voltage for Case 1 is shown in Fig. 8. Numerous peaks are evident in the spectrum between frequencies of 200 to $1000 \mathrm{~Hz}$. There are also several smaller peaks present between 1000 and $2000 \mathrm{~Hz}$. The lower-frequency energy content (below $100 \mathrm{~Hz}$ ) is due to vibration of the hotwire probe, and is not included in the plot. According to linear theory, the frequency range between 200 to $1000 \mathrm{~Hz}$ is the range of amplified traveling crossflow frequencies for this case. Mode shapes are plotted for three of the peaks in the spectrum in Fig. 9. The mode shapes of the larger amplitude modes compare very well with mode shapes predicted by linear PSE (not shown). Phase speeds and wavelengths were also calculated for each mode using the fixed probe as a phase reference, and these fell within the band of amplified wavelengths. There is quite a broad band of modes present in this case, which is typical of natural transition. A complete summary of the crossflow modes present in this dataset is presented in Table 2. There are several traveling crossflow modes present which are comparable in amplitude to the stationary crossflow modes. Table 2 also lists the uncertainty in the calculated value of the wavenumber. This uncertainty was estimated using a 95\% confidence interval for the phase using the method of Jenkins and Watts ${ }^{17}$, as well as the uncertainty of the best fit line applied to the phase vs. z-location plot. 


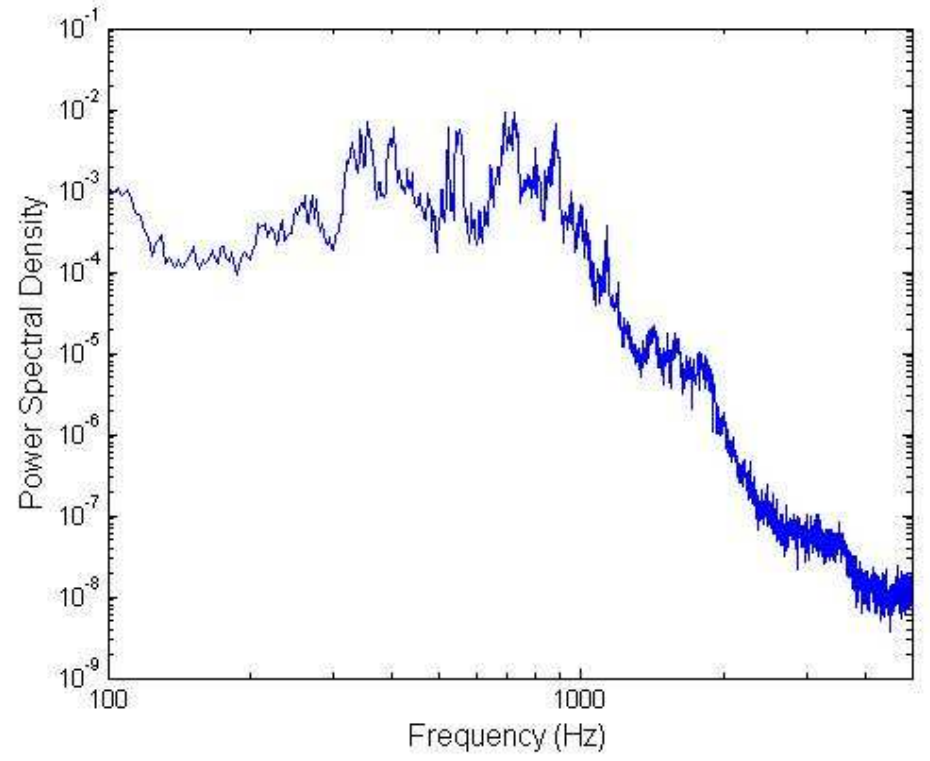

Figure 8. Auto-spectrum for Case 1

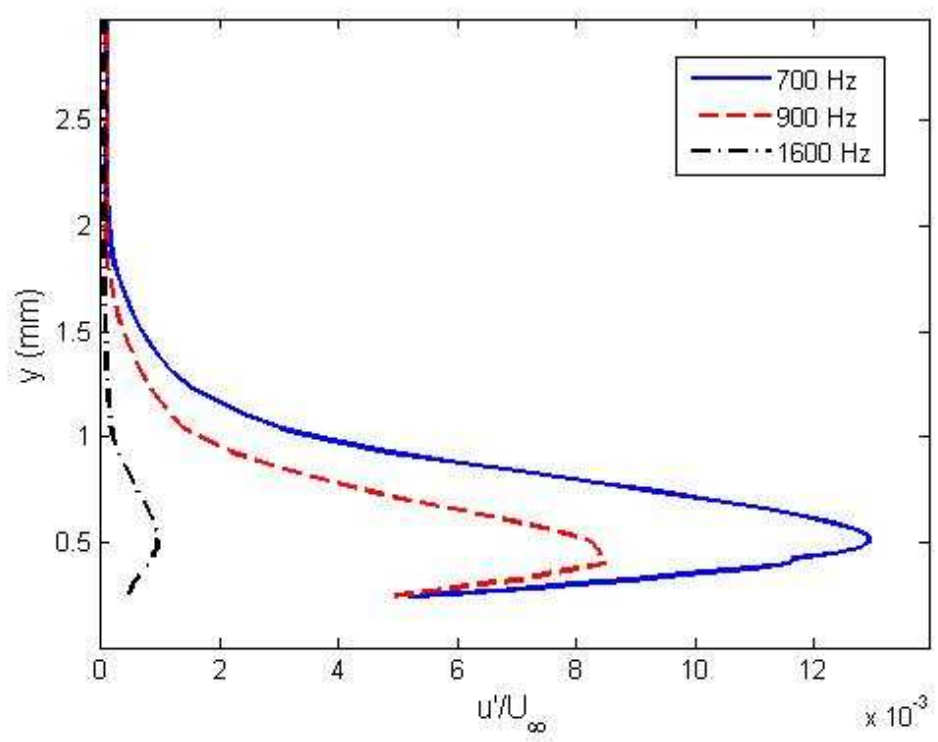

Figure 9. Traveling crossflow mode shapes for Case 1 
Table 2. Crossflow mode data for Case 1

\begin{tabular}{|c|c|c|c|c|c|}
\hline Peak Frequency $(\mathrm{Hz})$ & Frequency range $(\mathrm{Hz})$ & Amplitude $\left(\mathbf{u}^{\prime} / \mathbf{U}_{\infty}\right)$ & $\lambda(\mathrm{mm})$ & $\mathrm{k}\left(\mathrm{mm}^{-1}\right)$ & $\begin{array}{l}\text { Uncertainty } \\
\text { of } k\left(\mathrm{~mm}^{-1}\right)\end{array}$ \\
\hline 0 & 0 & 0.0184 & $10-20$ & - & - \\
\hline 0 & 0 & 0.02 & $5-10$ & - & - \\
\hline 350 & $300-400$ & 0.01 & 11.8 & 0.085 & \pm 0.0046 \\
\hline 550 & $500-600$ & 0.008 & 10.5 & 0.095 & \pm 0.0023 \\
\hline 700 & $600-750$ & 0.013 & 11.8 & 0.083 & \pm 0.0019 \\
\hline 900 & $820-920$ & 0.0085 & 14.1 & 0.071 & \pm 0.0017 \\
\hline 1250 & $1200-1300$ & 0.0008 & 4.90 & 0.204 & \pm 0.0121 \\
\hline 1400 & $1350-1450$ & 0.0008 & 5.84 & 0.171 & \pm 0.0095 \\
\hline 1600 & $1500-1700$ & 0.001 & 7.00 & 0.148 & \pm 0.0108 \\
\hline 1800 & $1700-1900$ & 0.0008 & - & - & - \\
\hline
\end{tabular}

\section{Resonant Triad Interaction}

The nonlinear interactions of the traveling crossflow modes were investigated by calculating the autobicoherence and the cross-bicoherence. The bicoherence $(b)$ is a measure of the phase locking between three frequencies in a time series such that $\theta\left(f_{l}\right) \pm \theta\left(f_{2}\right)=\theta\left(f_{l} \pm f_{2}\right)$. This triple phase-locking is one requirement for a triad resonance. The other requirement is wavenumber matching:

$$
k_{1} \pm k_{2}=k_{3}
$$

Kim and Powers ${ }^{18}$ showed that the square of the bicoherence is a measure of the power of the wave at $f_{3}$ due to the coupling of the waves at $f_{l}$ and $f_{2}$. This is true, at least, for a three-wave system, but the relationship may not be so simple for a broadband process. Bicoherence is a normalized quantity with values ranging from 0 to 1 , with 1 indicating perfect phase locking, and 0 representing no phase-locking. The bicoherence involves three frequencies: $f_{1}$ and $f_{2}$ are represented on the $\mathrm{x}$ and $\mathrm{y}$-axes respectively, and $f_{3}$ is represented as the sum of $f_{1}$ and $f_{2}$. Therefore, the value of bicoherence at a particular $\left(f_{1}, f_{2}\right)$ represents the interaction of the three waves at $f_{1}, f_{2}$, and $f_{3}=f_{1}+f_{2}$. The valid region of interacting frequencies forms a triangle (as in Fig. 10), due to the Nyquest frequency limit and symmetry conditions.

While the auto-bicoherence measures the phase-locking between three modes from a single time series, the cross-bicoherence is a measure of the mode interaction from two (or three) different time series. In this analysis, the cross-bicoherence is used to measure the interaction between $f_{1}$ and $f_{2}$ from the fixed probe time series, and $f_{3}$ from the traverse probe.

Even for a completely Gaussian process, the bispectrum for a finite-length time series will not be zero. Therefore, it is helpful to estimate a $95 \%$ significance level on zero bicoherence, which is given by Haubrich ${ }^{19}$ as

$$
b_{9 \mathrm{E} \% \mathrm{~b}}^{2} \geq 6 / \text { dof }
$$

where $d o f$ is the number of degrees of freedom. This means that there is $95 \%$ confidence that a calculated bicoherence above this value indicates a non-linear interaction (for a stationary and normally distributed process). This is actually a conservative estimate of the $95 \%$ significance level due to the different normalization used by Haubrich $^{19}$. In fact this equation yields a value closer to the $99 \%$ significance level for bicoherence calculated using the normalization of the present work ${ }^{20}$. For the bicoherence calculations in this paper, 512 degrees of freedom were used, which yields a 95\% significance level on zero bicoherence of 0.012 .

The squared auto-bicoherence for one data point (located near the wall-normal location of peak amplitude in the $700 \mathrm{~Hz}$ traveling crossflow mode shape) is shown in Fig. 10. This plot shows significant phase locking between several traveling crossflow modes. Three peaks are labeled in the figure. Peak 1 indicates an interaction between the $700 \mathrm{~Hz}$ mode and its harmonic at $1400 \mathrm{~Hz}(700 \mathrm{~Hz}, 700 \mathrm{~Hz})$ in the figure. Peak 2 indicates an interaction between two of the largest traveling crossflow modes $(700 \mathrm{~Hz}$ and $900 \mathrm{~Hz})$ and a mode at $1600 \mathrm{~Hz}$. Peak 3, which is actually two fairly distinct peaks, indicates an interaction between the $700 \mathrm{~Hz}, 1600 \mathrm{~Hz}$, and $2300 \mathrm{~Hz}$ modes, as well as an interaction between the $700 \mathrm{~Hz}, 1800 \mathrm{~Hz}$, and $2500 \mathrm{~Hz}$ modes. There also appears to be a lower amplitude peak at around $(900 \mathrm{~Hz}, 900 \mathrm{~Hz}$ ) (not labeled in the figure), indicating a harmonic at $1800 \mathrm{~Hz}$. 


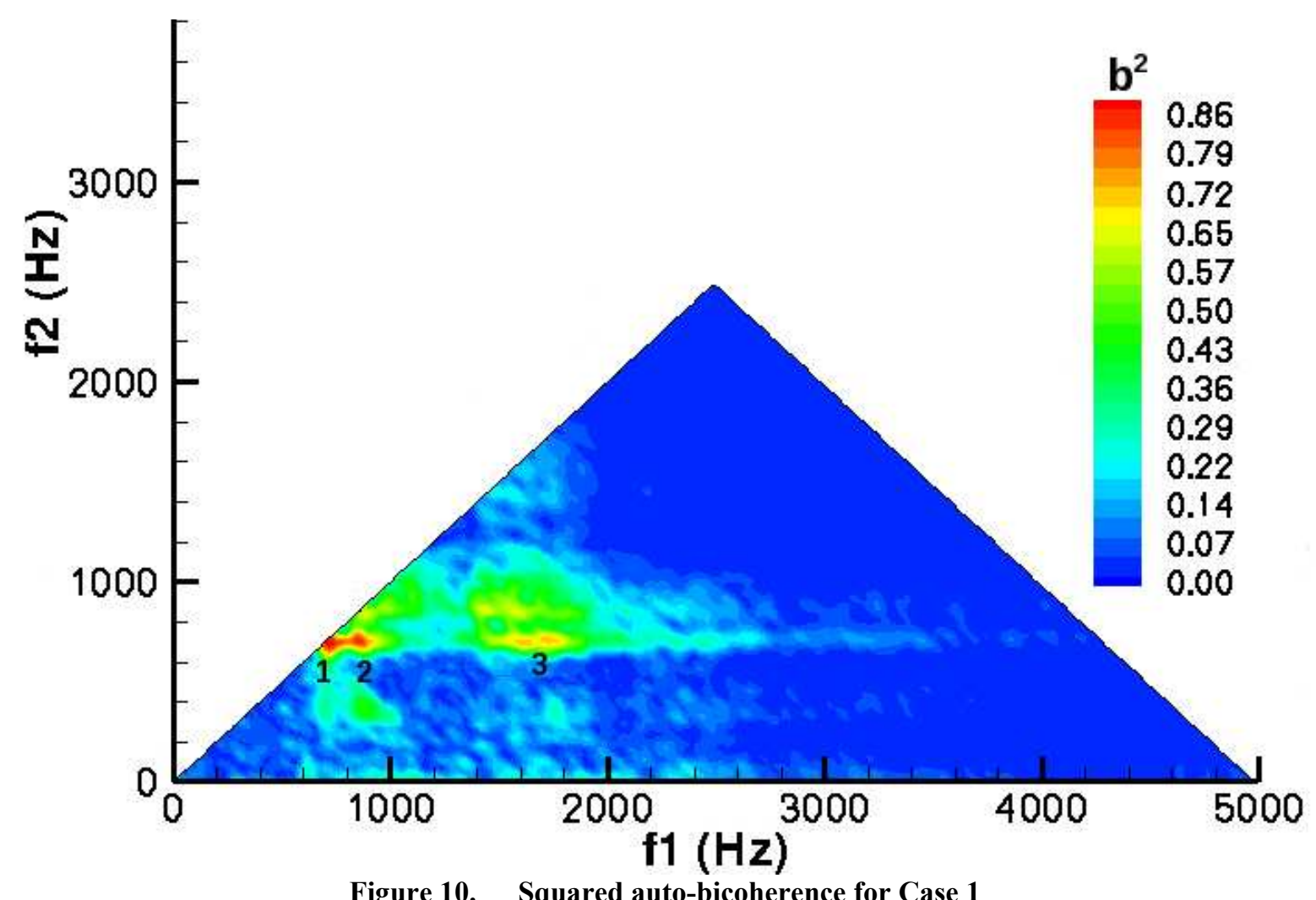

Figure 10. Squared auto-bicoherence for Case 1

The interaction of the $700 \mathrm{~Hz}$ and $900 \mathrm{~Hz}$ modes appears to be the strongest interaction in this case. In order for the resonance conditions to hold, the wavenumbers must satisfy Eq. (2). The wavenumbers for the three modes are listed in Table 2. In this case, $k_{f=700}+k_{f=900}=0.154 \mathrm{~mm}^{-1}$, and $k_{f=1600}=0.148 \mathrm{~mm}^{-1}$, which is reasonable agreement given the uncertainty of the wavenumber calculation. As indicated in Table 2, the amplitude of the $1600 \mathrm{~Hz}$ mode is an order of magnitude smaller than the traveling crossflow modes at $700 \mathrm{~Hz}$ and $900 \mathrm{~Hz}$. However, the fact that it is present at all is interesting, since the linear PSE results indicate that this mode $(f=1600 \mathrm{~Hz}, \lambda=7 \mathrm{~mm})$ is not unstable over any region of the airfoil. This suggests that the $1600 \mathrm{~Hz}$ mode is a result of the interaction between the two primary modes $(700 \mathrm{~Hz}$ and $900 \mathrm{~Hz}$ modes). Figure 9 shows the mode shapes of each of the interacting modes for this case. The shape of the $1600 \mathrm{~Hz}$ mode is actually quite similar to that of the $700 \mathrm{~Hz}$ and $900 \mathrm{~Hz}$ modes. The $(700 \mathrm{~Hz}, 700 \mathrm{~Hz})$ harmonic interaction (Peak 1 in Fig. 10) gives similar results. In this case, $k_{f=700}+k_{f=700}=0.166 \mathrm{~m}^{-1}$, and $k_{f=1400}=0.171 \mathrm{~mm}^{-1}$, which again matches within the estimated uncertainty. Once again, the amplitude of the higher frequency mode is an order of magnitude lower than the primary mode, and the linear PSE results for this mode $(f=1400 \mathrm{~Hz}, \lambda=6 \mathrm{~mm})$ indicate that it is stable over the entire airfoil.

As a way of investigating the fidelity of these results, the wall-normal phase profile of the $1600 \mathrm{~Hz}$ mode was calculated using several different methods. First, the phase profile was determined using the fixed probe as a reference and calculating the phase shift between the two $1600 \mathrm{~Hz}$ modes. Second, the phase profiles measured for the $700 \mathrm{~Hz}$ and $900 \mathrm{~Hz}$ modes (using the fixed probe as a reference) were used as input to determine the phase profile of the $1600 \mathrm{~Hz}$ mode assuming a purely multiplicative nonlinear interaction, so $\theta\left(f_{3}\right)=\theta\left(f_{1}\right)+\theta\left(f_{2}\right)$. Finally, the cross-biphase of the $1600 \mathrm{~Hz}$ mode was calculated, using the fixed probe for the 700 and $900 \mathrm{~Hz}$ frequencies, and the traverse probe for the $1600 \mathrm{~Hz}$ mode. The results for one spanwise location are shown in Fig. 11, and the shapes of the phase profiles exhibit remarkable agreement. 


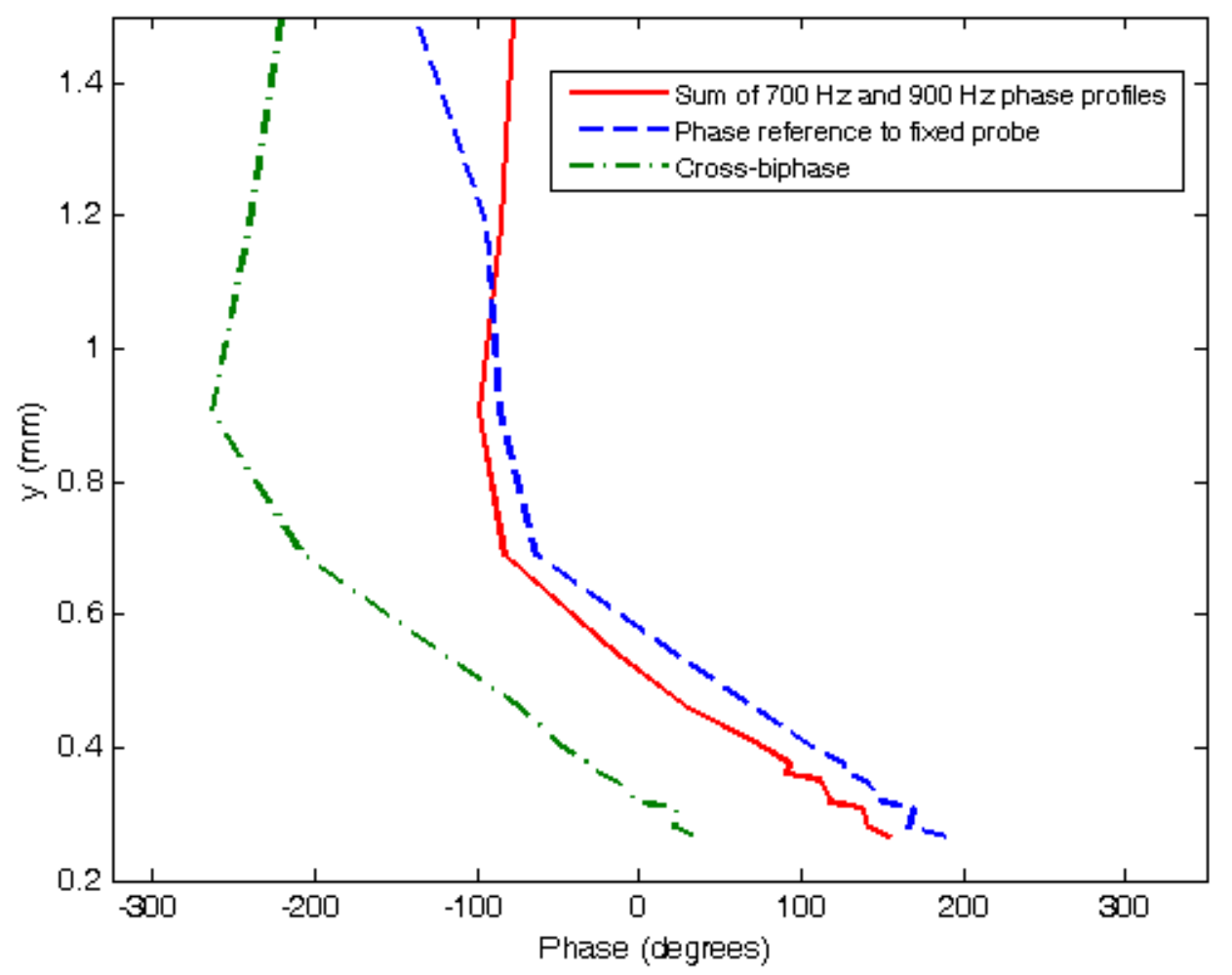

Figure 11. Comparison of phase profiles for the $1600 \mathrm{~Hz}$ mode calculated for Case 1

One interesting observation to be made from Fig. 11 is the phase shift between the phase profile calculated using the cross-biphase and the profile from the directly measured phase shift of the $1600 \mathrm{~Hz}$ modes (the green and blue lines respectively). The average phase shift over the entire phase profile is roughly 140 degrees. By examining the auto-biphase of the fixed probe, it is evident that this shift in phase is a result of the relative phase of the $1600 \mathrm{~Hz}$ mode at the fixed probe to the two interacting modes at the fixed probe. The auto-biphase of the traverse probe data reveals a similar phase shift. This indicates that the nonlinear interaction of the $700 \mathrm{~Hz}$ and $900 \mathrm{~Hz}$ modes creates a phase shift, but the physical mechanism is not yet understood.

Perhaps the most interesting (and most significant) observation to be made from the bicoherence plot (Fig. 10) relates to the two peaks labeled Peak 3 in the figure. These peaks indicate interactions between the 700,1600, and $2300 \mathrm{~Hz}$ modes and the 700, 1800, and $2500 \mathrm{~Hz}$ modes. This demonstrates that the higher frequency modes which were presumably created due to the interaction of the primary modes, now themselves interact with the primary modes to create even higher frequency modes. This very clearly implies a mechanism for spectral broadening, and subsequent breakdown to turbulence.

To further characterize the higher frequency mode $(2300 \mathrm{~Hz})$, we attempted to calculate a spanwise wavenumber in order to determine whether there is in fact wavenumber matching with the interacting modes. Unfortunately, this was not possible using the two-sensor coherence approach that is typically used, since the coherence at $2300 \mathrm{~Hz}$ with the fixed probe was too low to permit calculation of the phase. However, the cross-bicoherence between the 700,1600 , and $2300 \mathrm{~Hz}$ modes was large enough to permit a calculation of the phase speed. Using this approach, the spanwise wavenumber of the $2300 \mathrm{~Hz}$ mode was found to be $0.234 \pm 0.006 \mathrm{~mm}^{-1}$, while $k_{f=700}+k_{f=1600}=0.231$ $\mathrm{mm}^{-1}$ (from Table 2). Interestingly, there were two other modes that were interacting to create a $2300 \mathrm{~Hz}$ mode, namely the $900 \mathrm{~Hz}$ and $1400 \mathrm{~Hz}$ modes. When the phase speed was calculated in a similar fashion, but using the cross-biphase at $(900 \mathrm{~Hz}, 1400 \mathrm{~Hz})$ instead of at $(700 \mathrm{~Hz}, 1600 \mathrm{~Hz})$, the spanwise wavenumber was found to be $0.229 \pm 0.006 \mathrm{~mm}^{-1}$, while $k_{f=900}+k_{f=1400}=0.242 \mathrm{~mm}^{-1}$. In both cases, the wavenumber matching is quite satisfactory. It is also interesting to note that the phase profiles calculated using the cross-biphase for $(700 \mathrm{~Hz}, 1600 \mathrm{~Hz})$ and $(900$ $\mathrm{Hz}, 1400 \mathrm{~Hz}$ ) are very similar up to the edge of the mode shape (Fig. 12). Beyond the edge of the mode, the coherence is low. Apparently, two separate interactions were simultaneously feeding energy into the same mode. 


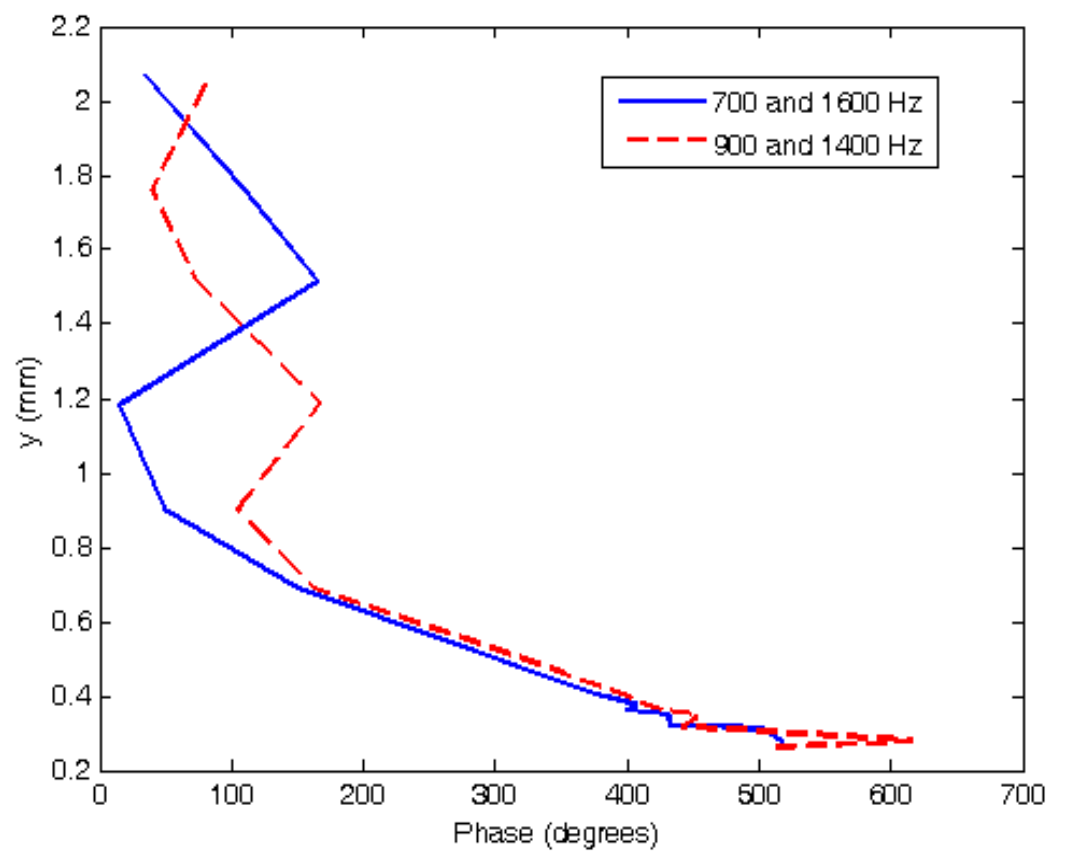

Figure 12. Comparison of phase profiles calculated using the cross-biphase for two sets of interacting modes

\section{Case 2}

1. Traveling Crossflow

A representative power spectrum for this case is shown in Fig. 13. As in the previous case, there are numerous peaks evident in the spectrum from $200 \mathrm{~Hz}$ to $1000 \mathrm{~Hz}$. The amplitudes of the largest traveling crossflow modes in this case are larger than those in the previous case (see Table 3), but are smaller in comparison to the stationary crossflow amplitude (which was around $6 \%$ for this case). However, they are still the same order of magnitude. An interesting thing to note from the spectrum is that the energy at the higher frequencies is much higher $\left(\sim 10^{-5}\right)$ than in Case $1\left(\sim 10^{-8}\right)$. Unfortunately, for this case, the coherence with the fixed probe was not consistently high enough to obtain phase speeds, so no spanwise wavelengths could be reliably calculated. A possible explanation is discussed below.

Table 3. Crossflow mode data for Case 2

\begin{tabular}{|c|c|c|}
\hline Peak Frequency (Hz) & Frequency range (Hz) & Amplitude $\left(\mathbf{u}^{\prime} / \mathbf{U}_{\infty}\right)$ \\
\hline 0 & 0 & 0.061 \\
\hline 230 & $170-280$ & 0.012 \\
\hline 550 & $530-620$ & 0.011 \\
\hline 690 & $670-740$ & 0.015 \\
\hline 770 & $740-825$ & 0.02 \\
\hline 880 & $860-900$ & 0.01 \\
\hline 980 & $900-1040$ & 0.015 \\
\hline 1320 & $1200-1400$ & 0.0065 \\
\hline 1750 & $1650-1850$ & 0.0043 \\
\hline
\end{tabular}




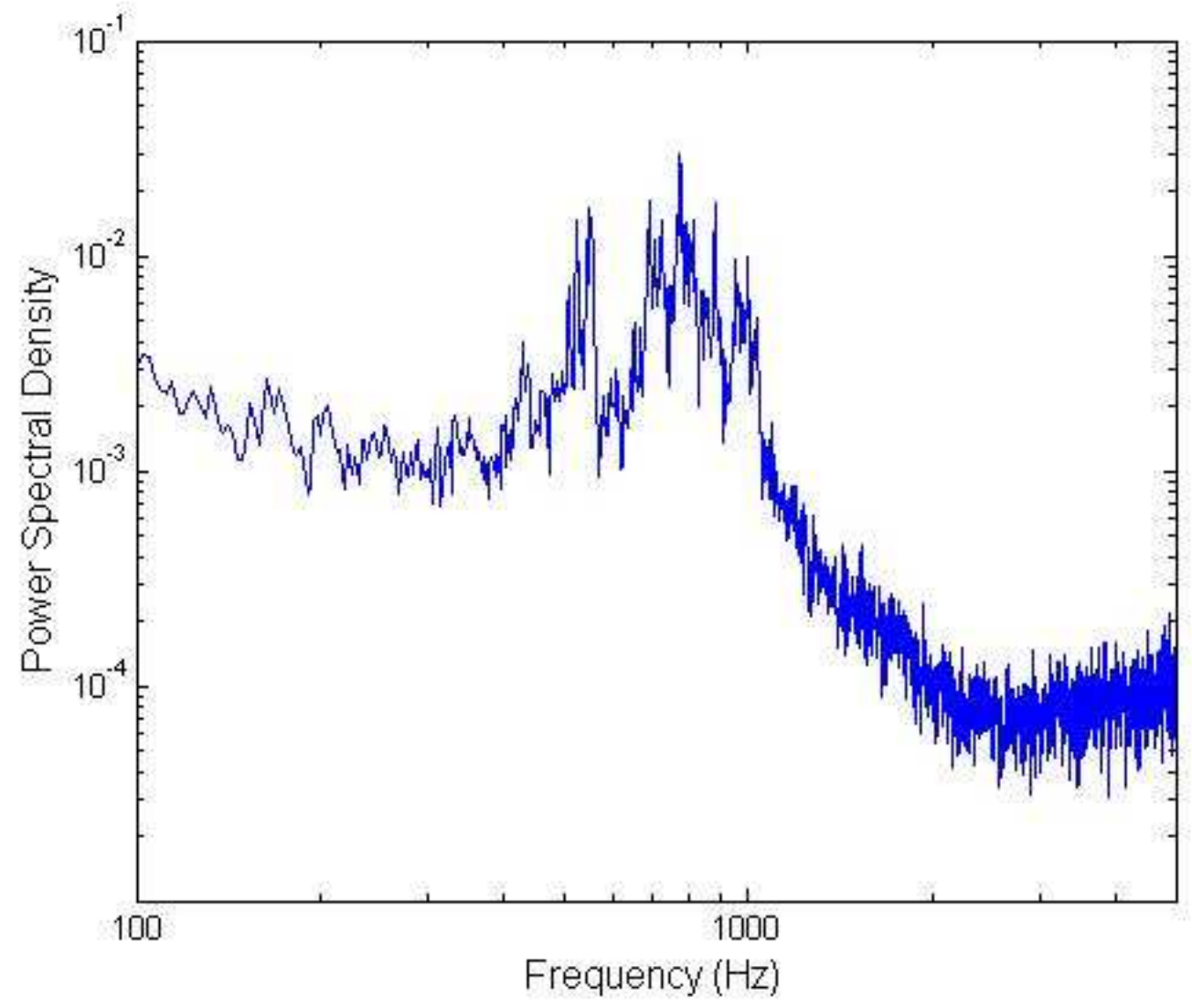

Figure 13. Auto-spectrum for Case 2

\section{Resonant Triad Interaction}

The auto-bicoherence for a representative point is shown in Fig. 14. This plot shows some similar interactions as in the previous case. The primary modes in this case are at slightly different frequencies $(770$ and $880 \mathrm{~Hz})$. The behavior is similar to the previous case in that there is an interaction between the two primary modes (labeled 2 in the plot), as well as harmonic interactions (labeled 1 and 3 in the plot). One obvious difference from the previous case is the apparent increase of spectral broadening. The primary modes evidently interact with numerous higher frequency modes. Given that this dataset was taken closer to the transition location than the previous case, the evidence points again to a spectral broadening mechanism leading to transition.

As mentioned earlier, the coherence between the fixed probe and the traverse probe was too low and inconsistent across the span to calculate a spanwise wavenumber. Unfortunately, the same is true of the cross-bicoherence, and therefore this method also could not be used. Based on prior evidence from the auto-bicoherence results, it is reasonable to assume that the bicoherence plot in this case is the result of triad resonance interactions among the traveling crossflow modes. The lack of consistency in the coherence across the span in this case is likely due to the large number of interacting modes, and the fact that this dataset was taken closer to the transition location. 


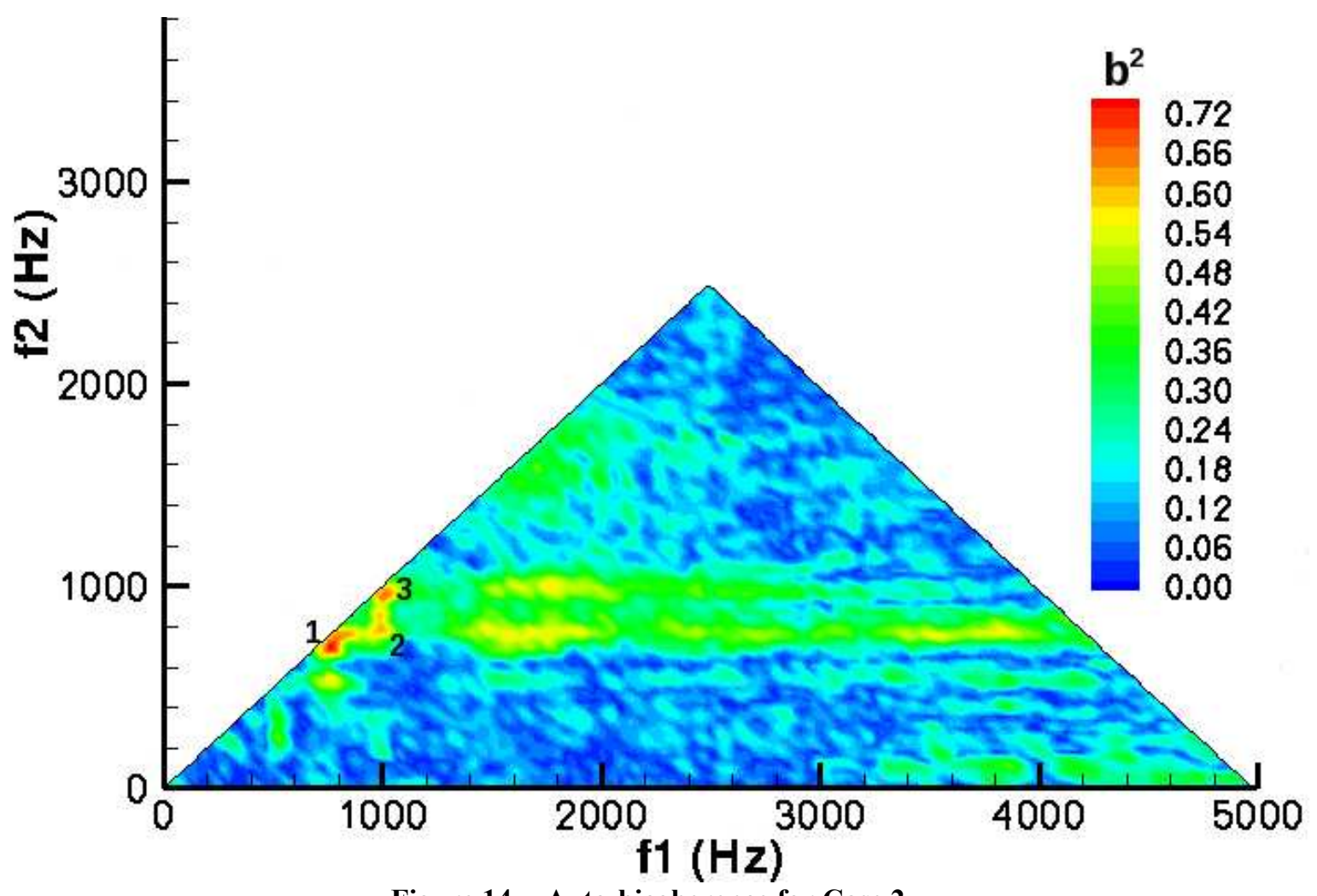

Figure 14. Auto-bicoherence for Case 2

\section{Conclusion and Discussion}

Non-linear resonant triad interactions among traveling crossflow modes were observed for two specific cases in the NASA/Boeing HLFC Experiment. This is the first evidence of this interaction on a swept wing, and it may have important implications for laminar flow wind tunnel testing as well as laminar flow control technology for swept wings. In both cases for which resonant interaction was observed, two primary linearly unstable modes interact to produce a higher frequency mode at the sum of their frequencies. There are also harmonic interactions for both of the primary modes. In each case, the higher frequency mode is linearly stable over the entire airfoil.

Perhaps the most important observation from this data analysis is the spread of resonant interactions to increasingly high frequencies. The higher frequency modes created from the initial interaction of the primary modes interact with the primary modes, and this in turn spreads energy to even higher frequencies. Such spectral broadening presumably continues until transition occurs.

Based on the limited amount of data currently available, some hypotheses can be made about this type of interaction. In such cases, the stationary crossflow and primary traveling crossflow amplitudes were on the order of $1-6 \%$ of $U_{\infty}$, so they were subcritical and of the same order of magnitude. At this point, it is unclear what role the stationary crossflow modes play in this mechanism. It is also unclear what the selection mechanism is for this interaction (i.e. what causes certain modes to interact while others do not). It seems probable that this type of interaction requires a broad band of unstable modes, such as that normally found in natural transition. However, this interaction may be difficult to recreate by forcing the unstable modes.

The possible implications of these results are two-fold. Since initial amplitudes of traveling crossflow modes are linked to the freestream turbulence level, stationary crossflow is assumed to dominate in flight environments while traveling crossflow may be more important in wind tunnel testing. Therefore, this type of breakdown mechanism might make it impossible to obtain accurate results in laminar flow wind tunnel testing, even for the quietest wind tunnels. Even if one can successfully damp the stationary crossflow modes, the mechanism of interacting traveling crossflow modes may lead to early transition. On the other hand, this mechanism may be important in flight where the freestream turbulence levels are low. More data is required in order to determine the necessary conditions for this mechanism to occur because it is possible that it may exist at very low amplitudes of the traveling crossflow 
instability. Even if one is able to design a wing which can successfully damp stationary crossflow modes, the resonance mechanism may lead to premature transition.

\section{Acknowledgments}

The authors would like to gratefully acknowledge the input and guidance of Drs. Meelan Choudhari, Craig Streett, and Mujeeb Malik, NASA Langley Research Center. This work is supported by the Subsonic Fixed Wing Project of the Fundamental Aeronautics Program.

\section{References}

${ }^{1}$ Klebanoff, P. S., Tidstrom, K. D., and Sargent, L. M., "The Three-Dimensional Nature of Boundary-Layer Instability," Journal of Fluid Mechanics, Vol. 12, 1962, pp. 1-34.

${ }^{2}$ Craik, A. D. D., "Non-linear Resonant Instability in Boundary Layers,” Journal of Fluid Mechanics, Vol. 50, 1971, pp. 393413.

${ }^{3}$ Herbert, T., "Subharmonic Three-Dimensional Disturbances in Unstable Plane Shear Flows," AIAA Paper No. 83-1759, 1983.

${ }^{4}$ Saric, W. S. and Thomas, A. S. W., "Experiments on the Subharmonic Route to Turbulence in Boundary Layers,"

Turbulence and Chaotic Phenomena in Fluids, edited by T. Tatsumi, North-Holland, 1984, pp.117-122.

${ }^{5}$ Lekoudis, S. G., "Resonant Wave Interactions on a Swept Wing," AIAA Journal, Vol. 18, 1980, pp. 122-124.

${ }^{6}$ Malik, M. R., Li, F., and Chang, C. -L., "Crossflow Disturbances in Three-Dimensional Boundary Layers: Nonlinear Development, Wave Interaction and Secondary Instability," Journal of Fluid Mechanics, Vol. 268, 1994, pp. 1-36.

${ }^{7}$ Corke, T. C. and Knasiak, K. F., "Stationary Travelling Crossflow Mode Interactions on a Rotating Disk," Journal of Fluid Mechanics, Vol. 355, 1998, pp. 285-315.

${ }^{8}$ Dagenhart, J. R., Saric, W. S., Mousseux, M. C., and Stack, J. P., "Crossflow-Vortex Instability and Transition on a 45degree Swept Wing," AIAA Paper No. 89-1892, 1989.

${ }^{9}$ Wlezien, R., Eppink, J., and Cronin, C., "Web-based Interactive Wind Tunnel Testing - A Historical Perspective," AIAA Paper No. 2010-4213, 2010.

${ }^{10}$ Choudhari, M. M., Li, F., Chang, C. -L., and Malik, M. R., "NASA/Boeing HLFC Experiment: Data Analysis With Advanced CFD Tools," High Technology Report No. HTC-9607, 1996.

${ }^{11}$ High Technology Corporation, "NASA/Boeing HLFC Experiment: Data Analysis,” NAS1-20267, 1996.

${ }^{12}$ Street, C. L., "Designing a Hybrid Laminar-Flow Control Experiment - the CFD-Experiment Connection," AIAA Paper No. 2033-979, 2003.

${ }^{13}$ Eppink, J. and Wlezien, R., "Data Analysis for the NASA/Boeing Hybrid Laminar Flow Control Crossflow Experiment," AIAA Paper No. 2011-3879, 2011.

${ }^{14}$ Radetsky, R. H., Reibert, M. S., Saric, W. S., and Takagi, S., "Effect of Micron-Sized Roughness on Transition in SweptWing Flows," AIAA Paper No. 93-0076, 1993.

${ }^{15}$ Bippes, H., "Basic Experiments on Transition in Three-Dimensional Boundary Layers Dominated by Crossflow Instability," Progress in Aerospace Sciences, Vol. 35, 1999, pp. 363-412.

${ }^{16}$ Radetsky, R. H., Reibert, M. S., and Saric, W. S., "Development of Stationary Crossflow Vortices on a Swept Wing," AIAA Paper No. 94-2373, 1994.

${ }^{17}$ Jenkins, G. M. and Watts, D. G., Spectral Analysis and Its Applications, Holden-Day, San Francisco, CA, 1968.

${ }^{18}$ Kim, Y. C. and Powers, E. J., "Digital Bispectral Analysis and its Applications to Nonlinear Wave Interactions," IEEE Trans. Plasma Science, Vol. 1, 1979, pp. 120-131.

${ }^{19}$ Haubrich, R. A., "Earth Noises, 5 to 500 Millicycles per Second," J. Geophys. Res., Vol. 70, 1965, pp. 1415-1427.

${ }^{20}$ Elgar, S. and Guza, R. T., "Observations of Bispectra of Shoaling Surface Gravity Waves,” J. Fluid Mech., Vol. 161, 1985, p. $425-448$ 